\title{
Rats Fed Diets with Different Energy Contribution from Fat Do Not Differ in Adiposity
}

\author{
Alexander D. Miras ${ }^{a} \quad$ Florian Seyfried $^{b} \quad$ Alkystis Phinikaridou $^{c}$ \\ Marcelo E. Andiac, d Ioannis Christakis ${ }^{a}$ Alan C. Spector ${ }^{\mathrm{e}}$ \\ René M. Botnar ${ }^{c, f, g}$ Carel W. le Roux ${ }^{a}, h$ \\ a Section of Investigative Medicine, Imperial College London, UK; ${ }^{b}$ Department of General \\ and Visceral, Vascular and Pediatric Surgery, University of Wurzburg, Wurzburg, Germany; \\ 'Division of Imaging Sciences and Biomedical Engineering, The Rayne Institute, 4th Floor, \\ Lambeth Wing, St. Thomas' Hospital, King's College London, London, UK; ${ }^{d}$ Radiology \\ Department, School of Medicine, Pontificia Universidad Catolica de Chile, Santiago, \\ Chile; ${ }^{e}$ Department of Psychology and Program in Neuroscience, Florida State University, \\ Tallahassee, FL, USA; ${ }^{f}$ British Heart Foundation Centre of Excellence, Cardiovascular Division, \\ King's College London, London, UK; ' ${ }^{2}$ Wellcome Trust and EPSRC Medical Engineering

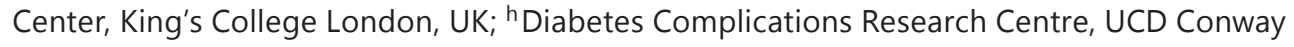 \\ Institute, School of Medicine and Medical Science, University College Dublin, Dublin, Ireland
}

\section{Key Words}

High-fat diet · Low-fat diet · MRI - Leptin

\section{Abstract}

Objective: To determine whether rats reaching the same body mass, having been fed either a low-fat (LFD) or a high-fat diet (HFD), differ in white adipose tissue (WAT) deposition. Methods: In experiment 1, 22 Sprague-Dawley rats of the same age were divided into 11 rats with body mass below the batch median and fed a HFD, and 11 above the median and fed a LFD. In experiment 2, 20 Sprague-Dawley rats of the same age and starting body mass were randomised to either a HFD or LFD. When all groups reached similar final body mass, WAT was quantified using magnetic resonance imaging (MRI), dissection, and plasma leptin. Results: In experiment 1, both groups reached similar final body mass at the same age; in experiment 2 the HFD group reached similar final body mass earlier than the LFD group. There were no significant differences in WAT as assessed by MRI or leptin between the HFD and LFD groups in both experiments. Dissection revealed a trend for higher retroperitoneal and epididymal adiposity in the HFD groups in both experiments. Conclusions: We conclude that at similar body mass, adiposity is independent of the macronutrient composition of the feeding regimen used to achieve it. 
Miras et al.: Rats Fed Diets with Different Energy Contribution from Fat Do Not Differ in Adiposity

\section{Introduction}

The sharp rise in the incidence of obesity and its associated co-morbidities has led to intense investigation of the pathophysiological processes that result from or cause this disease state. Animal models of obesity have attracted considerable interest as they allow researchers to perform behavioural, genetic or physiological manipulations and to study their effects using more invasive methodologies in a short period of time, without the social and cognitive confounders of human experimentation. The consumption of calorically dense high-fat diets (HFDs) and carbohydrate-rich diets is considered as one of the major factors in the development of obesity [1]. Animals rendered obese through dietary or genetic manipulation are therefore good models to study the physiological derangements of the condition [2].

The term 'diet-induced obesity' is often defined as 'HFD-induced obesity' due to the overwhelming evidence that animals fed diets high in fat (and energy), gain more mass and have higher proportion of total body and visceral white adipose tissue (WAT) compared to animals fed a low-fat (and energy) diet (LFD) for the same period of time [2, 3]. However, there are significant differences in the phenotype, body composition and metabolic profiles of animals fed HFDs amongst the published studies $[2,4]$. These differences are probably due to the various animal models studied including strain, age, gender, type of dietary fatty acids, macronutrient contribution and palatability [2,4]. It is also unclear whether it is the total energy intake or the percentage energy contribution from dietary fat that has greater contribution on WAT accumulation [5]. Indeed, in obesity clinics it is recognised that some patients develop obesity through the consumption of high volumes of low-fat food [6, 7]. A relatively small number of studies have investigated the effects of diets containing different amounts of fat on the adiposity of animals reaching the same body mass [8-14]. Dissection and chemical analyses techniques have yielded incongruent results, with the majority of studies showing that dietary fat content has a greater effect on adiposity compared to total energy consumption per se $[8,10-14]$, and others showing the reverse [9].

In this study we performed two experiments in an attempt to clarify these discrepancies. In the first experiment we controlled for the confounding factor of age and body mass at sacrifice, and in the second experiment we controlled for starting body mass (and therefore obesity proneness) upon introduction of the high-fat diet and body mass at sacrifice. In experiment 1 , we studied rats of the same initial age and batch, but of different body mass. The rats with a body mass below the median were fed a HFD and the rats with a mass above the median were fed a LFD. The two groups therefore reached the same final body mass at the same age. In experiment 2 , we studied rats of the same initial age, batch and body mass. One group was randomised to a HFD and the other to a LFD. The high-fat fed animals therefore reached the same final body mass earlier than the low-fat fed animals. When all 4 groups reached similar final body mass, we quantified WAT deposition using dissection, plasma leptin measurements and magnetic resonance imaging (MRI) technology. We hypothesised that even at similar final body mass, the adiposity of the high-fat fed animals would be higher than the adiposity of the low-fat fed animals in both experiments and wanted to quantify the magnitude.

\section{Material and Methods}

Animals and Diets

42 male Sprague-Dawley rats (Harlan, UK) were single housed under a 12/12 h light/dark cycle at room temperature $\left(21 \pm 2{ }^{\circ} \mathrm{C}\right)$. Water and solid food were available ad libitum. In experiment 1,22 animals aged 10 weeks were divided into two groups of 11 rats above and below and median for body mass. The rats below the median body mass of $337.0 \mathrm{~g}$ had free access to a HFD (C1090-60, Altromin GmbH \& Co. KG, Germany, 


\begin{tabular}{l|l}
\hline Obes Facts 2014;7:302-310 \\
\hline DOI: $10.1159 / 000368622$ & $\begin{array}{l}\text { () 2014 S. Karger GmbH, Freiburg } \\
\text { www.karger.com/ofa }\end{array}$ \\
\hline
\end{tabular}

Miras et al.: Rats Fed Diets with Different Energy Contribution from Fat Do Not Differ in Adiposity

Table 1. Diet composition ${ }^{\mathrm{a}}$

\begin{tabular}{|c|c|c|}
\hline \multirow{2}{*}{$\begin{array}{l}\text { Macronutrient and detailed } \\
\text { composition }\end{array}$} & \multicolumn{2}{|c|}{$\%$ of total energy contribution } \\
\hline & $\begin{array}{l}\text { HFD } \\
(5,017.1 \mathrm{kcal} / \mathrm{kg})\end{array}$ & $\begin{array}{l}\text { LFD } \\
(3,520.6 \mathrm{kcal} / \mathrm{kg})\end{array}$ \\
\hline Protein & 17.0 & 17.5 \\
\hline Lysine & 1.0 & 0.7 \\
\hline Methionine & 0.6 & 0.2 \\
\hline Cystine & 0.1 & 0.2 \\
\hline Threonine & 0.5 & 0.5 \\
\hline Tryptophan & 0.1 & 0.2 \\
\hline Arginine & 1.0 & 0.9 \\
\hline Histidine & 0.3 & 0.4 \\
\hline Isoleucine & 0.6 & 0.5 \\
\hline Leucine & 1.0 & 1.0 \\
\hline Phenylalanine & 0.6 & 0.7 \\
\hline Valine & 0.7 & 0.7 \\
\hline Alanine & 1.0 & 0.2 \\
\hline Aspartic acid & 1.0 & 0.7 \\
\hline Glutamic acid & 2.3 & 3.2 \\
\hline Glycine & 1.7 & 1.1 \\
\hline Proline & 1.6 & 1.2 \\
\hline Serine & 0.7 & 0.6 \\
\hline Tyrosine & 0.4 & 0.5 \\
\hline Carbohydrates & 23.0 & 75.1 \\
\hline Mono and disaccharides & 19.6 & 4.9 \\
\hline Fat & 60 & 7.4 \\
\hline \multicolumn{3}{|l|}{ Saturated fatty acids } \\
\hline C-10:0 capric acid & 0.6 & $\mathrm{n} / \mathrm{a}$ \\
\hline C-12:0 lauric acid & 0.6 & 0.0 \\
\hline C-14:0 myristic acid & 0.3 & 0.1 \\
\hline $\mathrm{C}-15: 0$ pentadecanic acid & 0.2 & $\mathrm{n} / \mathrm{a}$ \\
\hline C-16:0 palmitic acid & 16.4 & 0.3 \\
\hline C-18:0 stearic acid & 8.6 & 0.01 \\
\hline \multicolumn{3}{|l|}{ Monounsaturated fatty acids } \\
\hline C-16:1 palmitoleic acid & 1.7 & 0.1 \\
\hline C-18:1 oleic acid & 19.2 & 0.8 \\
\hline \multicolumn{3}{|l|}{ Polyunsaturated fatty acids } \\
\hline C-18:2 linoleic acid & 5.2 & 0.7 \\
\hline C-18:3 linolenic acid & 0.3 & 0.1 \\
\hline C-20:2 eicosadienoic acid & 0.2 & $\mathrm{n} / \mathrm{a}$ \\
\hline C-20:4 arachidonic acid & 0.0 & 0.1 \\
\hline
\end{tabular}

aThe table shows the percentage energy contribution of the nutrients in the two diets used in the two experiments

non-purified; table 1) while the rats above the body mass median of 337.0 g received a LFD (RM1, diet Special Diet Services Ltd, UK, non-purified; table 1). In experiment 2, 20 animals aged 10 weeks were randomised to either a HFD $(n=10)$ or a LFD $(n=10)$ independently of their body mass. Body mass and food intake were assessed at the onset of the light cycle.

\section{Body Composition Assessment}

When all animals reached a similar final body mass of 534-544 g, they were sacrificed in the fasted state through cardiac puncture under anaesthesia with $5 \%$ isoflurane and $2 \%$ oxygen. Blood was collected in EDTA tubes and centrifuged at $3,000 \mathrm{rpm}$ for $10 \mathrm{~min}$ at $4{ }^{\circ} \mathrm{C}$. The supernatant was stored in a $-80{ }^{\circ} \mathrm{C}$ freezer until 
Miras et al.: Rats Fed Diets with Different Energy Contribution from Fat Do Not Differ in Adiposity

further analysis. Rats were scanned in batches of 2-4 at a time in a clinical 3 Tesla MR scanner (Achieva; Philips Healthcare, Best, the Netherlands) using a 32-element cardiac receiver coil. Animals were scanned in the supine position and were kept at room temperature. To identify the abdomen, liver and heart, a fast multistack (transverse, sagittal and coronal), multi-slice, two-dimensional gradient echo sequence was performed. Imaging parameters included field-of-view $(\mathrm{FOV})=350 \mathrm{~mm}$, matrix $=300 \times 150$, in-plane resolution $=1.2 \times$ $2.3 \mathrm{~mm}$, repetition time $/$ echo time $(\mathrm{TR} / \mathrm{TE})=13.9 / 1.3 \mathrm{~ms}$, flip angle $=50^{\circ}$, slice thickness $=10 \mathrm{~mm}$ and 1 signal average. Subsequently, a two-point DIXON 3D gradient echo scan was planned in the coronal plane to cover the entire abdomen and thorax [15]. Using this chemical shift imaging technique a water-only and fat-only dataset can be produced from one dual-echo acquisition. This allows quantification of the water and fat content of tissues in the imaging volume. Coronal images were acquired with: FOV $=380 \times 380 \mathrm{~mm}$, matrix $=340 \times 340$, in-plane resolution $=1 \times 1 \mathrm{~mm}, \mathrm{TR}=5.8 \mathrm{~ms}, \mathrm{TE}_{1}=3.1 \mathrm{~ms}$ and $\mathrm{TE}_{2}=4.3 \mathrm{~ms}$, flip angle $=25^{\circ}$, slice thickness $=2 \mathrm{~mm}$ and signal averages $=3$. MRI volumetric analysis of the fat was performed by a single investigator blinded to the experimental protocol, using the free open source image analysis software MITK 3M3 (German Cancer Research Center Mint Medical GmbH, Heidelberg, Germany). The analysis included the whole animal, but not its tail. Total body WAT volume $\left(\mathrm{cm}^{3}\right)$ was converted to mass (grams) by multiplying with $0.92 \mathrm{~g} / \mathrm{cm}^{3}$, the assumed density of WAT [16].

Immediately following MRI scanning, the retroperitoneal and epididymal WAT pads were dissected according to a standardised protocol $[17,18]$ by one researcher who was blinded to the feeding regimen used. A midline laparotomy was performed, the intestine was removed, and retroperitoneum was exposed. The aorta was considered as the medial, the fascia transversalis the lateral, and the psoas muscle as the dorsal dissection border. The retroperitoneal and epididymal WAT pads were dissected and weighed using a precision balance. We did not assess the mesenteric and inguinal fat as we have found their quantification through dissection to be highly variable. All experiments were performed in accordance to UK Home Office regulations under the project licence (PL 70-6669).

Leptin Measurements

Plasma leptin was measured using a mouse leptin ELISA Quantikine kit from R \& D Systems Europe Ltd (Abington, UK).

\section{Statistical Methodology}

Comparisons of all the variables between the groups of each experiment were made through the use of unpaired Student's t tests or with ANCOVA with final body mass as a covariate. The body mass throughout the study was compared using repeated measures two-way ANOVA. In order to test the wider validity of these results, comparisons between all 4 groups of both experiments were also performed using a one-way ANOVA or ANCOVA with final body mass as a covariate. Correlations were made using Pearson's correlation testing (data shown with $\mathrm{r}$ representing the Pearson's correlation coefficient and $\mathrm{p}$ the statistical significance of the correlations). Statistics software: GraphPad Prism ${ }^{\circledR}$, version 5 (GraphPad Software, Inc., La Jolla, CA, USA).

\section{Results}

\section{Body Mass and Food Intake}

As per the design of experiment 1, the mean starting mass of the HFD fed rats was significantly lower than that of the LFD fed rats. HFD animals gained significantly more mass compared to LFD fed rats whether expressed in absolute terms or as a percentage of their starting mass. This was due to a significantly higher caloric intake in the HFD group. After 6 weeks there was no significant difference between the final body mass of the groups (fig. 1).

As per the design of experiment 2, there were no significant differences between the HFD and LFD groups in terms of their starting body mass, final body mass and body mass gain. The HFD group consumed significantly more calories per day compared to the LFD group, and it took 4.5 and 6 weeks for the HFD and LFD rats, respectively, to achieve their final body mass (table 2). 
Miras et al.: Rats Fed Diets with Different Energy Contribution from Fat Do Not Differ in Adiposity

Table 2. Summary of results*

\begin{tabular}{|c|c|c|c|c|c|c|c|}
\hline & \multicolumn{3}{|l|}{ Experiment 1} & \multicolumn{3}{|l|}{ Experiment 2} & \multirow{2}{*}{$\begin{array}{l}\text { Overall p value } \\
\text { Post hoc pair-wise } \\
\text { comparisons }\end{array}$} \\
\hline & $\begin{array}{l}\mathrm{HFD}_{1} \text { group } \\
(\mathrm{n}=11)\end{array}$ & $\begin{array}{l}\mathrm{LFD}_{1} \text { group } \\
(\mathrm{n}=11)\end{array}$ & $\mathrm{p}$ value & $\begin{array}{l}\mathrm{HFD}_{2} \text { group } \\
(\mathrm{n}=10)\end{array}$ & $\begin{array}{l}\mathrm{LFD}_{2} \text { group } \\
(\mathrm{n}=10)\end{array}$ & $\mathrm{p}$ value & \\
\hline Starting body mass, $\mathrm{g}$ & $320.4 \pm 3.1$ & $346.2 \pm 2.5$ & $<0.0001^{\mathrm{a}}$ & $326.4 \pm 4.4$ & $324.6 \pm 3.9$ & $0.76^{\mathrm{a}}$ & 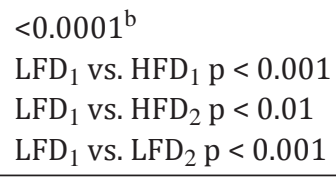 \\
\hline Final body mass, $g$ & $544.2 \pm 8.6$ & $538.5 \pm 3.3$ & $0.58^{\mathrm{a}}$ & $527.9 \pm 11.8$ & $534.6 \pm 12.6$ & 60.70 & $0.70^{\mathrm{b}}$ \\
\hline $\begin{array}{l}\text { Absolute increase in body } \\
\text { mass, } \mathrm{g}\end{array}$ & $223.8 \pm 9.9$ & $192.3 \pm 5.7$ & $0.01^{\mathrm{a}}$ & $201.5 \pm 8.0$ & $210.0 \pm 9.2$ & $0.50^{\mathrm{a}}$ & $0.06^{\mathrm{b}}$ \\
\hline $\begin{array}{l}\text { Relative increase in body } \\
\text { mass, \% of starting mass }\end{array}$ & $70.1 \pm 3.4$ & $55.6 \pm 1.8$ & $0.001^{\mathrm{a}}$ & $61.6 \pm 1.9$ & $64.5 \pm 2.2$ & $0.33^{\mathrm{a}}$ & $\begin{array}{l}0.002^{\mathrm{b}} \\
\mathrm{LFD}_{1} \text { vs. } \mathrm{HFD}_{1} \mathrm{p}<0.001\end{array}$ \\
\hline Caloric intake, kcal/day & $125.1 \pm 2.0$ & $113.4 \pm 1.8$ & $0.002^{\mathrm{a}}$ & $132.0 \pm 3.2$ & $118.7 \pm 2.9$ & $0.007^{\mathrm{a}}$ & $\begin{array}{l}<0.001^{\text {b }} \\
\mathrm{LFD}_{1} \text { vs. } \mathrm{HFD}_{1} \mathrm{p}<0.01 \\
\mathrm{HFD}_{1} \text { vs. } \mathrm{HFD}_{2} \mathrm{p}<0.05 \\
\mathrm{LFD}_{2} \text { vs. } \mathrm{HFD}_{2} \mathrm{p}<0.01\end{array}$ \\
\hline $\begin{array}{l}\text { Final total WAT, g } \\
\text { (quantified with MRI) }\end{array}$ & $121.2 \pm 11.7$ & $104.7 \pm 8.0$ & $0.33^{\mathrm{c}}$ & $136.3 \pm 11.0$ & $119.9 \pm 7.7$ & $0.19^{\mathrm{c}}$ & $0.07^{\mathrm{c}}$ \\
\hline $\begin{array}{l}\text { Final retroperitoneal and } \\
\text { epididymal WAT, g } \\
\text { (quantified with } \\
\text { dissection) }\end{array}$ & $22.5 \pm 1.4$ & $18.6 \pm 1.2$ & $0.07^{\mathrm{C}}$ & $25.2 \pm 1.1$ & $21.4 \pm 1.8$ & $0.06^{\mathrm{c}}$ & $\begin{array}{l}0.008^{\mathrm{c}} \\
\mathrm{LFD}_{1} \text { vs. } \mathrm{HFD}_{2} \mathrm{p}=0.005\end{array}$ \\
\hline $\begin{array}{l}\text { Final plasma leptin levels, } \\
\mathrm{ng} / \mathrm{ml}\end{array}$ & $7.4 \pm 0.5$ & $7.1 \pm 0.4$ & $0.57^{\mathrm{C}}$ & $8.9 \pm 0.9$ & $7.5 \pm 1.0$ & $0.29^{\mathrm{C}}$ & $0.21^{\mathrm{c}}$ \\
\hline
\end{tabular}

*The table summarises the main results of experiments 1 and 2. Data are expressed as mean \pm standard error of the mean (SEM).

As indicated by superscript letters, statistical comparisons have been performed between the 2 groups within experiments 1 and 2 using ${ }^{a}$ unpaired $t$ tests or ${ }^{c}$ ANCOVA with final total body mass as the covariate, but also between all 4 groups using ${ }^{b} a$ one-way ANOVA or ${ }^{\mathrm{C} A N C O V A}$ with final total body mass as the covariate and post hoc pair-wise Bonferonni corrected testing.

Subscripts: 1 -from experiment 1 , 2 -from experiment 2 .

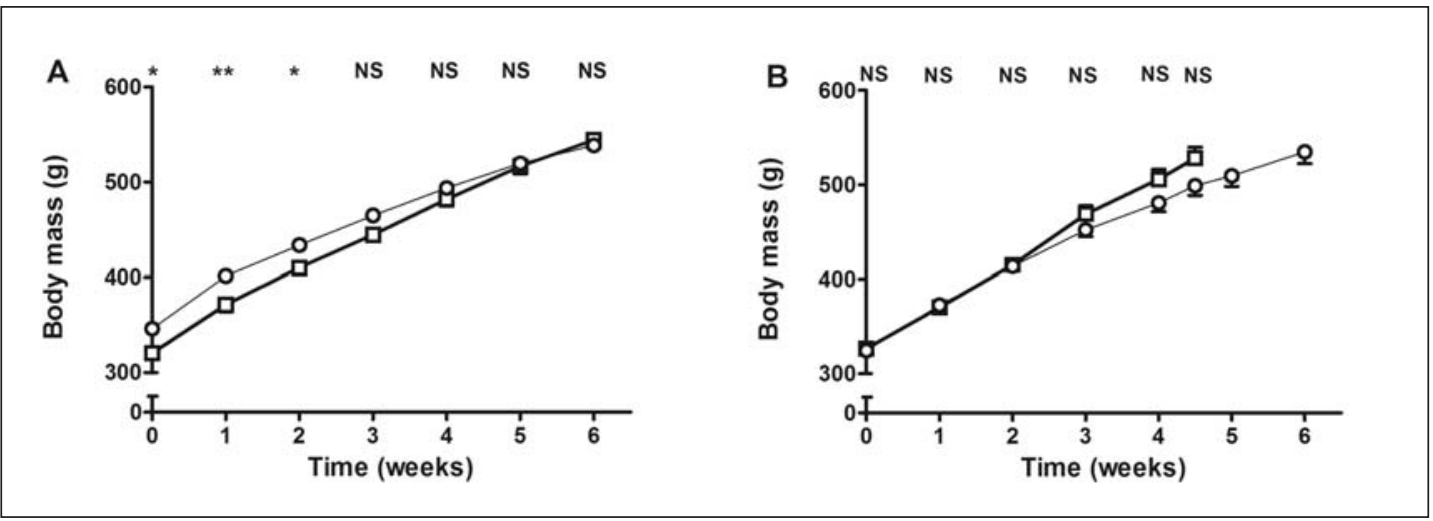

Fig. 1. Curves of the body mass for the high-fat diet (squares) fed and low-fat diet fed (circles) rat groups along the duration of experiment 1 (A, $n=11$ per group) and experiment 2 (B, n = 10 per group). Levels of significance: ${ }^{*} \mathrm{p}<0.05 * * \mathrm{p}<0.01$. NS = Not significant. 
Miras et al.: Rats Fed Diets with Different Energy Contribution from Fat Do Not Differ in Adiposity

\section{Total WAT Quantified by MRI}

Total WAT, adjusted for final total body mass, was not different between the HFD and LFD groups in experiments 1 and 2 (table 2).

\section{Retroperitoneal and Epididymal WAT Quantified by Dissection}

In both experiments, the difference in retroperitoneal and epididymal WAT mass adjusted for final total body mass (in grams) between the groups did not reach significance, although there was a trend for the HFD fed rats to having more retroperitoneal and epididymal adiposity. Dissected retroperitoneal and epididymal WAT correlated with total WAT quantified by MRI in each group (Experiment 1 : HFD group $r=0.67, p=0.024 ;$ LFD group $r=0.74$, $\mathrm{p}=0.009$; Experiment 2: HFD group $\mathrm{r}=0.68, \mathrm{p}=0.032$; LFD group $\mathrm{r}=0.68, \mathrm{p}=0.031$ ) (table 2).

\section{Leptin}

There was no significant difference in plasma leptin concentrations, adjusted for final total body mass, between the HFD and LFD groups in experiments 1 and 2. Leptin concentrations did not correlate with total or retroperitoneal and epididymal WAT mass in the groups (table 2).

\section{Discussion}

In this study we have shown that Sprague-Dawley rats that reached the same final body mass had similar total body WAT composition independently of whether they were fed a HFD or LFD. Whilst in our study the dynamic of body mass gain was different between the groups, with HFD fed rats gaining mass at a faster rate than the LFD rats (as expected), when the groups reached similar final total body mass, they did not differ in WAT deposition as assessed by dissection, MRI and leptin measurements. We used MRI methodology in view of its high sensitivity, reliability, non-invasive nature and because its measurements correlate very well with the gold standard chemical composition methodologies [16, 19-21].

The few studies that attempted to address the same question have yielded incongruent results. Storlien et al. [10] studied 16 male Wistar rats pair-fed either a $59 \%$ or $10 \%$ (\% energy contribution) fat diet for approximately 3 weeks ( $\mathrm{n}=8$ in each group). The investigators used a chemical composition analysis and showed that, even though the two groups had similar final total body mass, the percentage of total body adipose tissue was significantly higher in the high-fat fed group $(18.2 \pm 0.9 \%$ vs. $13.8 \pm 0.8 \%)$. The HFD fed group was also more insulin resistant and had lower post-prandial energy expenditure compared to the LFD fed group.

Boozer et al. [8] studied 4 groups of male Sprague-Dawley rats ( $\mathrm{n}=9-10$ per group) fed isocalorically one of four diets: 12\% (control diet), 24\%, 36\% and 48\% fat. The protein composition of the diets was constant, whereas the carbohydrate composition was decreased the higher the fat composition of the diet. The three experimental groups were pair fed in amounts equal to match the mean caloric consumption of the control group for 6 weeks in order reach similar final body mass. Using chemical composition analysis, the investigators found that the total body fat increased proportionally to the fat composition of their diet, with mesenteric fat increasing disproportionally. Despite the adiposity differences, plasma glucose and insulin levels after a fixed meal were similar between the groups.

Shiraev et al. [11] used a similar paradigm in Sprague-Dawley rats, but quantified WAT through dissection. Even though their results are in line with those from Storlien et al. [10] and Boozer et al. [8], the absolute value of total white fat mass dissected was remarkably low $(<8 \%)$. 
Miras et al.: Rats Fed Diets with Different Energy Contribution from Fat Do Not Differ in Adiposity

Woods et al. [9] studied male Long-Evans rats, including an ad libitum HFD fed group, an ad libitum LFD fed group and a group pair fed HFD isocalorically to the ad libitum LFD fed rats for approximately 10 weeks ( $\mathrm{n}=12-16$ per group). The precise energy contribution of macronutrients in the two purified diets was not detailed in the methods, but the body composition was quantified with a chemical composition method. As expected, the HFD fed group achieved higher total body and adipose tissue mass compared to both the LFD and the HFD pair fed groups. However, similar to our findings, the total body mass and the percentage of total body adipose tissue did not differ between the ad libitum LFD fed and the HFD pair fed group.

Considering that the majority of the literature in the field suggests that dietary fat content has a greater effect on adiposity compared to energy consumption per se, we were intrigued with the lack of any significant differences in final adiposity. It is noteworthy that the HFD in our study contained more sugars than the LFD, an additional difference in macronutrients that would have been expected to magnify differences in final adiposity (e.g. [22, 23]). Even though our sample size and duration of feeding was similar to that of other published studies, our dissection results did not show large differences as hypothesized, but only a trend for a difference in retroperitoneal and epididymal adiposity between the HFD and LFD fed groups in both experiments.

The discrepancies between the results of the first three studies $[8,10,11]$ with Woods et al. [9] and our study may be due to a number of factors. There was variation in the rat strains used and the duration of the feeding protocols. The nature and palatability of the fat used in the diets may also have contributed to the different final adiposity mass achieved, as for example rats fed vegetable-derived fat diets accumulate less adipose tissue compared to animal fed isoenergetic animal-derived fat diets [24]. The body composition quantification methods used in the other studies are based either on chemical analyses that are not identical to each other or on dissection which can yield variable results [19]. More importantly, as noted in the study by Woods et al. [9], it is not possible to exclude that food restriction, a food-deprived state and a meal pattern of feeding, all a potential result of pairfeeding in all the above experiments, could have influenced total adipose tissue accumulation [25].

Other groups have attempted to answer the same question using different paradigms. In a series of experiments on the metabolic and gender specific effects of high-fat feeding, Llado et al. [12-14] fed rats with a high-fat (and energy) or low-fat (and energy) diet, but by altering their protein/carbohydrate ratio managed to avoid hyperphagia in the high-fat fed rats. As a result, both the HFD and LFD fed groups consumed the same calories and reached the same final mass. Adiposity was assessed by dissection and suggested that HFD feeding resulted in higher WAT accumulation in male but not female rats. In a few other studies, rodents fed HFD and/or high-sugar diets isocalorically to the control groups, who were fed a LFD, gained more mass and more adiposity compared to the control group, even without differences in energy expenditure between the two groups [26-28]. The authors suggested that, when fed HFD, rats become more efficient in storing it. Finally, only one study reported that rats with ad libitum access to a HFD exhibited no hyperphagia, consumed fewer calories and still developed more adiposity compared to the LFD fed control group [29].

A limitation of the current study was that, whilst the diets used were chosen based on their fat content, they were provided by two manufacturers and were not made by purified components. The dissection of the WAT did not include subcutaneous fat, because of the poor reproducibility of dissection as a measurement for subcutaneous fat. We also did not assess total body WAT at baseline or measure fat within tissues (e.g. liver, muscle). Finally, we did not compare the two groups in terms of eating behaviour or more detailed metabolic parameters. 
Miras et al.: Rats Fed Diets with Different Energy Contribution from Fat Do Not Differ in Adiposity

In conclusion, we have shown in two complementary experiments that rats that achieve similar body mass through exposure to diets of different fat composition do not differ in measures of adiposity, even when controlling for the confounding effects of age or starting body weight upon the introduction of feeding. We are not questioning the important metabolic dysfunction caused by high-fat feeding. Our study does not exclude differences in metabolic parameters between the groups studied, but rather focussed specifically on adiposity. Under these circumstances, rats rendered equally 'obese' through a HFD or LFD could be used in obesity studies where for example the animals may be less able to tolerate HFDs such as after Roux-en-Y gastric bypass surgery [30].

\section{Acknowledgements}

We would like to thank Mr Marcus Ritter for his help with the analysis of the MRI images. A.D.M has received funding from a MRC Clinical Training Fellowship and a MRC Centenary Award, C.W.IR. from the Science Foundation Ireland /YI/B2480 and the Moulton Charitable Foundation, and F.S. from the Deutsche Forschungsgemeinschaft (F.S., SE 2027/1-1). The Section is funded by grants from the MRC, BBSRC, NIHR, an Integrative Mammalian Biology (IMB) Capacity Building Award, an FP7-HEALTH-2009-241592 EuroCHIP grant and is supported by the NIHR Imperial Biomedical Research Centre Funding Scheme. The funders did not have any role in the design, collection, analysis and interpretation of data, writing of the manuscript as well as in the decision to submit the manuscript for publication.

\section{Authors' Contributions}

C.W.IR. and A.C.S. designed research, A.D.M., F.S., A.P., M.E.A. and I.C. conducted research, R.M.B. provided essential materials, A.D.M. analysed data, A.D.M. wrote the paper, C.W.IR. had had primary responsibility for final content. A.D.M. and F.S. contributed equally to this manuscript.

\section{Disclosure Statement}

On behalf of all authors, the corresponding author states that there is no conflict of interest.

\section{References}

1 Bray GA, Popkin BM: Dietary fat intake does affect obesity! Am J Clin Nutr 1998;68:1157-1173.

2 Hariri N, Thibault L: High-fat diet-induced obesity in animal models. Nutr Res Rev 2010;23:270-299.

-3 Sclafani A, Springer D: Dietary obesity in adult rats: similarities to hypothalamic and human obesity syndromes. Physiol Behav 1976;17:461-471.

4 Buettner R, Scholmerich J, Bollheimer LC: High-fat diets: modeling the metabolic disorders of human obesity in rodents. Obesity (Silver Spring) 2007;15:798-808.

5 Bray GA, Smith SR, de Jonge L, Xie H, Rood J, Martin CK, Most M, Brock C, Mancuso S, Redman LM: Effect of dietary protein content on weight gain, energy expenditure, and body composition during overeating: a randomized controlled trial. JAMA 2012;307:47-55.

6 Willett WC: Is dietary fat a major determinant of body fat? Am J Clin Nutr 1998;67(3 suppl):556S-562S.

7 Moussavi N, Gavino V, Receveur 0: Could the quality of dietary fat, and not just its quantity, be related to risk of obesity? Obesity (Silver Spring) 2008;16:7-15.

8 Boozer CN, Schoenbach G, Atkinson RL: Dietary fat and adiposity: a dose-response relationship in adult male rats fed isocalorically. Am J Physiol 1995;268:E546-550.

9 Woods SC, Seeley RJ, Rushing PA, D'Alessio D, Tso P: A controlled high-fat diet induces an obese syndrome in rats. J Nutr 2003;133:1081-1087.

10 Storlien LH, James DE, Burleigh KM, Chisholm DJ, Kraegen EW: Fat feeding causes widespread in vivo insulin resistance, decreased energy expenditure, and obesity in rats. Am J Physiol 1986;251:E576-583.

11 Shiraev T, Chen H, Morris MJ: Differential effects of restricted versus unlimited high-fat feeding in rats on fat mass, plasma hormones and brain appetite regulators. J Neuroendocrinol 2009;21:602-609. 
Miras et al.: Rats Fed Diets with Different Energy Contribution from Fat Do Not Differ in Adiposity

12 Catala-Niell A, Estrany ME, Proenza AM, Gianotti M, Llado I: Skeletal muscle and liver oxidative metabolism in response to a voluntary isocaloric intake of a high fat diet in male and female rats. Cell Physiol Biochem 2008; 22:327-336.

13 Estrany ME, Proenza AM, Llado I, Gianotti M: Isocaloric intake of a high-fat diet modifies adiposity and lipid handling in a sex dependent manner in rats. Lipids Health Dis 2011;10:52.

14 Nadal-Casellas A, Proenza AM, Llado I, Gianotti M: Sex-dependent differences in rat hepatic lipid accumulation and insulin sensitivity in response to diet-induced obesity. Biochem Cell Biol 2012;90:164-172.

15 Ma J: Dixon techniques for water and fat imaging. J Magn Reson Imaging 2008;28:543-558.

16 Tang H, Vasselli JR, Wu EX, Boozer CN, Gallagher D: High-resolution magnetic resonance imaging tracks changes in organ and tissue mass in obese and aging rats. Am J Physiol Regul Integr Comp Physiol 2002; 282:R890-899.

17 Gerbaix M, Metz L, Ringot E, Courteix D: Visceral fat mass determination in rodent: validation of dual-energy $\mathrm{x}$-ray absorptiometry and anthropometric techniques in fat and lean rats. Lipids Health Dis 2010;9:140.

18 Lac G, Cavalie H, Ebal E, Michaux 0: Effects of a high fat diet on bone of growing rats. Correlations between visceral fat, adiponectin and bone mass density. Lipids Health Dis 2008;7:16.

19 Johnson DH, Flask CA, Ernsberger PR, Wong WC, Wilson DL: Reproducible MRI measurement of adipose tissue volumes in genetic and dietary rodent obesity models. J Magn Reson Imaging 2008;28:915-927.

20 Ishikawa M, Koga K: Measurement of abdominal fat by magnetic resonance imaging of OLETF rats, an animal model of NIDDM. Magn Reson Imaging 1998;16:45-53.

-21 Ross R, Leger L, Guardo R, De Guise J, Pike BG: Adipose tissue volume measured by magnetic resonance imaging and computerized tomography in rats. J Appl Physiol 1991;70:2164-2172.

22 Sheludiakova A, Rooney K, Boakes RA: Metabolic and behavioural effects of sucrose and fructose/glucose drinks in the rat. Eur J Nutr 2012;51:445-454.

-23 Glendinning JI, Gillman J, Zamer H, Margolskee RF, Sclafani A: The role of T1r3 and Trpm5 in carbohydrateinduced obesity in mice. Physiol Behav 2012;107:50-58.

24 Shimomura Y, Tamura T, Suzuki M: Less body fat accumulation in rats fed a safflower oil diet than in rats fed a beef tallow diet. J Nutr 1990;120:1291-1296.

25 Cohn C, Joseph D: Effects on metabolism produced by the rate of ingestion of the diet. Am J Clin Nutr 1960;8: 682-690.

-26 Lomba A, Milagro FI, Garcia-Diaz DF, Campion J, Marzo F, Martinez JA: A high-sucrose isocaloric pair-fed model induces obesity and impairs NDUFB6 gene function in rat adipose tissue. J Nutrigenet Nutrigenomics 2009;2: 267-272.

27 Lomba A, Martinez JA, Garcia-Diaz DF, Paternain L, Marti A, Campion J, Milagro FI: Weight gain induced by an isocaloric pair-fed high fat diet: a nutriepigenetic study on FASN and NDUFB6 gene promoters. Mol Genet Metab 2010;101:273-278.

28 Lomba A, Milagro FI, Garcia-Diaz DF, Marti A, Campion J, Martinez JA: Obesity induced by a pair-fed high fat sucrose diet: methylation and expression pattern of genes related to energy homeostasis. Lipids Health Dis 2010;9:60.

29 Oscai LB, Miller WC, Arnall DA: Effects of dietary sugar and of dietary fat on food intake and body fat content in rats. Growth 1987;51:64-73.

-30 le Roux CW, Bueter M, Theis N, Werling M, Ashrafian H, Lowenstein C, Athanasiou T, Bloom SR, Spector AC, Olbers T, Lutz TA: Gastric bypass reduces fat intake and preference. Am J Physiol Regul Integr Comp Physiol 2011;301:R1057-1066. 